\title{
COLLABORATIVE Wi-Fi FINGERPRINTING INDOOR POSITIONING USING NEAR RELATION
}

\author{
Yankun Wang ${ }^{1 *}$, Weixi Wang ${ }^{1}$, Xiaoming $\mathrm{Li}^{1}{ }^{1}$, Wei Zhang ${ }^{1}$, Renzhong Guo ${ }^{1}$ \\ ${ }^{1}$ National Engineering Laboratory for Big Data System Computing Technology \& Guangdong Key Laboratory of Urban Informatics \\ \& Shenzhen Key Laboratory of Spatial Smart Sensing and Services \& Research Institute for Smart Cities, School of Architecture and \\ Urban Planning, Shenzhen University, Shenzhen, PR China - yankunwang@szu.edu.cn; wangwx@ szu.edu.cn; \\ lxmingster@163.com; weizhsz@szu.edu.cn; guorz2013@qq.com
}

Commission IV, WG IV/5

KEY WORDS: Indoor Positioning, Near relation, Sparse Wi-Fi Fingerprint, Fingerprint Ambiguity

\begin{abstract}
:
Indoor positioning is of great importance to the era of mobile computing. Currently, much attention has been paid to RSS-based location for that it can provide position information without additional equipment. However, this method suffers from many challenges: (1) fingerprint ambiguity; (2) labor-intensive of fingerprint collection; (3) low efficiency of fingerprint matching. To get over these drawbacks, we provide a collaborative WiFi fingerprinting indoor positioning method using near relation. The base idea of this method is that interpolation method is used to enrich sparse Wi-Fi fingerprint. Near relation boundary is provided and Wi-Fi fingerprints is constrained to this region to reduce fingerprint ambiguity, which also can improve the efficiency of fingerprint matching. Extensive experiments show that a positioning accuracy of $3.8 \mathrm{~m}$ can be achieved with the near relation under $1 \mathrm{~m}$ interpolation density.
\end{abstract}

\section{INSTRUCTION}

With the increasing popularity of mobile and pervasive computing, the location service based on indoor positioning has attracted much attention due to its wide applications in living, production, commence, and public services (Xia et al., 2017; Hu et al., 2018). Recent years have witnessed a lot of work on indoor positioning. Single source location (e.g., received signal strength, radio frequency identification, ultra wideband and visible light) and multi-source location based indoor positioning strategy are differentiated by the inference techniques such as the time of arrival, the time difference of arrival and the angle of arrival (Mainetti et al., 2014; Tang et al., 2019; Ren et al., 2019; Leca et al., 2020). RSS-based location has attracted much attention and is regard as one of the most promising solution for indoor positioning, for that it can provide position information without additional hardware and deploying investment (Brena et al., 2017; Zafari et al., 2017).

RSS-based localization mainly contains two stages: offline and online (Ge et al., 2016; Chen et al., 2017; Han et al., 2019). During the offline stage, the RSS fingerprints dataset mapping relationship between signal fingerprints and spatial locations is established. Next in the online stage, estimating location by matching the online RSS fingerprint collection with the offline fingerprint dataset (Du et al., 2107; Subedi et al., 2019). However, the RSS-based method faces many challenges: (1) Fingerprint ambiguity due to the multi-path reflection of RSS in indoor complex environment, which result two different locations may have similar RSS fingerprints and low positioning accuracy (Huang et al., 2019). (2) Fingerprint collection is labor-intensive and time-consuming (Hu et al., 2018). (3) Low efficiency in fingerprint database matching (Huang et al., 2019).

The literature on fingerprinting-based indoor localization is long and rich. The major reason of ambiguity issues come from two aspects: position ambiguity and RSS ambiguity (Han et al., 2015) The ambiguity of RSS can be reduced by adding the fingerprints dimension ( $\mathrm{Li}$ et al., 2016), using scene information (Liu et al., 2017) or PDR (Chen et al., 2015) to narrow the fingerprint database. $\mathrm{Li}$ ( $\mathrm{Li}$ et al., 2016) extended the fingerprints dimension from one point to multiple points to avoid the ambiguity of RSS. A multi-dimensional dynamic time warping (MD-DTW) algorithm is provided for matching. Liu (Liu et al., 2017) used the camera of a smartphone as "eye" to recognize the environment. With scene information constrained, the searching range of fingerprint database is narrowed down and RSS ambiguities are decreased. Chen (Chen et al., 2015) used pedestrian dead-reckoning (PDR) to track user movements and restricted the fingerprints to a circle center at the PDR positioning result. The interpolation method is widely used to encrypted fingerprint (Wang et al., 2017; Shen et al., 2019;). Voronoi Tessellation (Lee et al., 2012), Inverse Distance Weighting (IDW) (Zuo et al., 2018; Li et al., 2018), and Delaunay (Jan et al., 2015) are well-known methods. Lee (Lee et al., 2012) refined the propagation model for each cell of the target area tessellated by a higher-order Voronoi diagram. This method can take into account the signal fading caused by walls and obstacles more accurately, but the location of access point (AP) must be obtained in advanced. The basic idea of IDW is to provide weight for the data points based on their distance to the estimated point. The closer is the known data point to the estimated point, the more weight it is (Moghtadaiee et al., 2019). The linear interpolation method is based on Delaunay triangulation. The IDW and linear interpolation algorithm are compared to estimate the missing RSS values (Talvitie et al., 2015).

To overcome the drawbacks of traditional RSS-based indoor positioning method, our paper used Delaunay-based interpolation method to enrich sparse Wi-Fi fingerprints, which reduce the labor of fingerprint collection. Near relation is provided to constrain the Wi-Fi fingerprints matching space, which not only reduce the fingerprint ambiguity, but also improve matching efficiency.

The contributions of our paper are as follow:

(1) We identify the opportunity of near relation to resolve the fingerprints ambiguity. To the best of our knowledge, it is the

* Corresponding author 
first work combing spatial relationship in semantic descriptions with fingerprints.

(2) A near relation-based indoor positioning under sparse Wi-Fi fingerprinting scheme is provided. IDW and Delaunay based interpolation method are adopt to encrypt the sparse Wi-Fi fingerprinting. Near relation region is provided to narrow the searching space of fingerprinting and resolve the fingerprinting ambiguity.

(3) To evaluate our method, an experiment is conducted and the result demonstrate that a positioning accuracy of $3.8 \mathrm{~m}$ is achievable with near relation constrain under $1 \mathrm{~m}$ interpolation density.

The remainder of this paper is organized as follows. Section 2 describes the system overview and method. Section 3 presents the related experiments. Section 4 summarizes this work and discusses future research directions.

\section{SYSTEM OVERVIEW AND METHOD}

\subsection{System overview}

Definition 1. RO (Reference Object): An RO is any feature with a name in a place description.

Generally, there are reference objects (ROs) and their related spatial relations (SRs; i.e., topology, direction and distance) and target objects (TO) in a place description. A TO is the place that is to be described or located. Take the place description "near LaoFX" as example. LaoFX is the RO (Reference Object). Additionally, ROs are polygons indoors due to scale.

The architecture of the proposed system is similar to traditional fingerprinting localization system which contains two stages: offline and online stage. In the offline stage, the near relation boundary and a fingerprint database should be built. In the online stage, the near relation for the RO (Reference Object) should be selected to narrow the search space of fingerprinting before positioning.
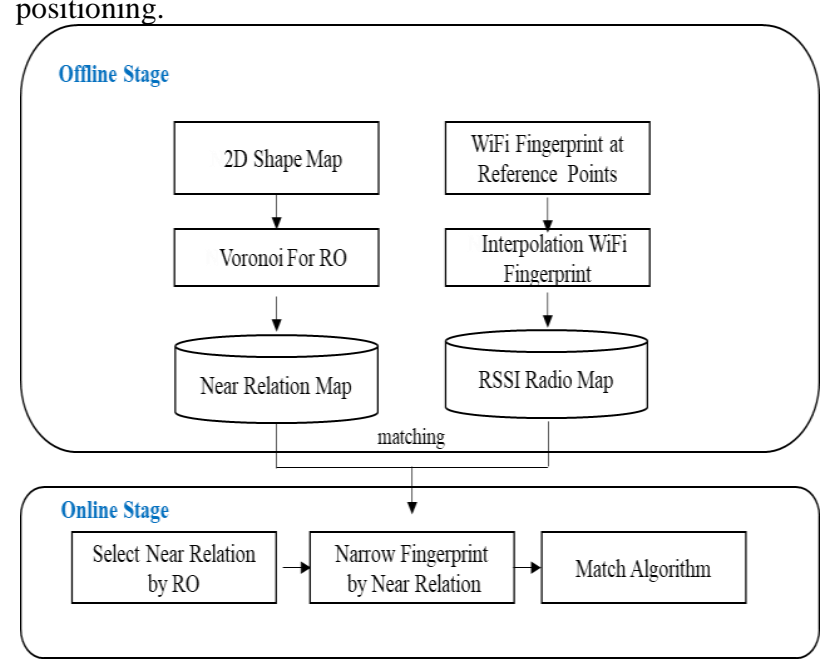

Figure 1. System overview.

\subsection{Method}

\subsubsection{Near relation boundary}

Definition 2. Voronoi region of RO: Let $\mathrm{R}\left(\mathrm{RO}_{1}, \mathrm{RO}_{2} \cdots \cdots \mathrm{RO}_{\mathrm{i}}\right)$ be a set of entities in the 2-D Euclidean plane $E^{2}$ and $d\left(p, R O_{i}\right)$ be the Euclidean distance between point $\mathrm{p}$ and $\mathrm{RO}_{\mathrm{i}}$. The minimum distance between $\mathrm{p}$ and $\mathrm{RO}_{\mathrm{i}}$ is denoted with $\mathrm{d}_{\min }\left(\mathrm{p}, \mathrm{RO}_{\mathrm{i}}\right)$. The Voronoi region of $\mathrm{RO}_{\mathrm{i}}$ is denoted with $\operatorname{Vor}\left(\mathrm{RO}_{\mathrm{i}}\right)$.

$\operatorname{Vor}\left(R O_{i}\right)=\left\{p \mid d_{\text {min }}\left(p, R O_{i}\right)<d_{\text {min }}\left(p, R O_{j}\right), p \in E^{2}, i \neq j\right\}(1)$

Definition 3. Neighbours of RO: The Voronoi regions of ROs which share the common edge neighbour each other. As shown in figure 2, The neighbours of $\mathrm{RO}_{1}$ is denoted with neigbor $\left(\mathrm{RO}_{1}\right)=\left\{\mathrm{RO}_{2}, \mathrm{RO}_{4}, \mathrm{RO} 5, \mathrm{RO}_{8}, \mathrm{RO}_{9} \mathrm{RO}_{10}\right\}$.

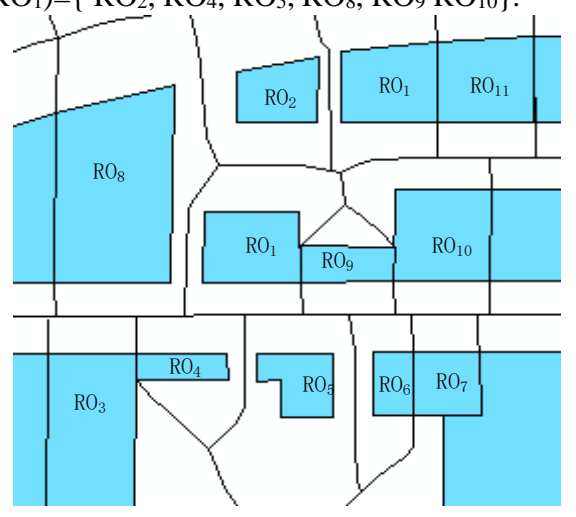

Figure 2. Neighbours of $\mathrm{RO}_{1}$

Definition 4. Near boundary of RO: The near boundary of RO refers to the region of a site insert into and neighbours to RO. The Delaunay triangulation is the "dual" of the Voronoi diagram. As shown in figure, the distances between vertex $\mathrm{V}_{2}$ to $\mathrm{RO}_{1}, \mathrm{RO}_{2}$ and $\mathrm{RO}_{7}$ are equal. Drawing the circles center on vertices of region of $\mathrm{RO}_{1}$ (i.e., $\mathrm{V}_{1}, \mathrm{~V}_{2}, \mathrm{~V}_{3}, \mathrm{~V}_{4}, \mathrm{~V}_{5}$ ). The nearboudary of $\mathrm{RO}_{1}$ not only consists of circle arcs but also the segments of its neighbours. The gray-dash line is the circles and the red solid region is the near boundary of $\mathrm{RO}_{1}$.

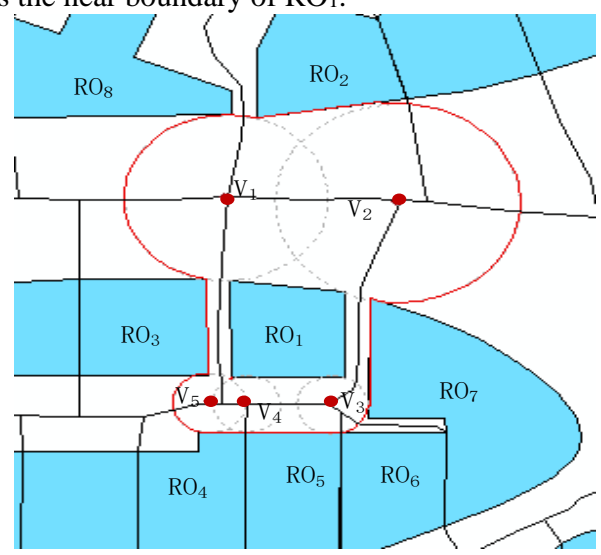

\subsubsection{Interpolation method}

Figure 3. Near boundary of $\mathrm{RO}_{1}$.

The interpolation method is based on Delaunay. After conducting the Delaunay triangle based on the known data, the entire interpolation process consists of interpolation and extrapolation. Interpolation and extrapolation refer to estimate the data inside and outside the Delaunay triangles respectively.

\section{A. Interpolation}

The first step is to determine in which triangle the interpolated point inside. After this, the value of interpolated data can be calculated as:

$$
P_{\text {interp }}=\omega_{1} P_{1}+\omega_{2} P_{2}+\omega_{3} P_{3}
$$

Where $\mathrm{P}_{1}\left(\mathrm{x}_{1}, \mathrm{y}_{1}\right), \mathrm{P}_{2}\left(\mathrm{x}_{2}, \mathrm{y}_{2}\right), \mathrm{P}_{3}\left(\mathrm{x}_{3}, \mathrm{y}_{3}\right)$ are the vertices of Delaunay triangle that $\mathrm{P}_{\text {interp }}(\mathrm{x}, \mathrm{y})$ inside in and $\omega_{1}+\omega_{2}+\omega_{3}=1$.

$$
\begin{gathered}
\omega_{1}=\frac{\left(y_{2}-y_{3}\right)\left(x-x_{3}\right)+\left(x_{3}-x_{2}\right)\left(y-y_{3}\right)}{\left(y_{2}-y_{3}\right)\left(x_{1}-x_{3}\right)+\left(x_{3}-x_{2}\right)\left(y_{1}-y_{3}\right)} \\
\omega_{2}=\frac{\left(y_{3}-y_{1}\right)\left(x-x_{3}\right)+\left(x_{1}-x_{3}\right)\left(y-y_{3}\right)}{\left(y_{2}-y_{3}\right)\left(x_{1}-x_{3}\right)+\left(x_{3}-x_{2}\right)\left(y_{1}-y_{3}\right)} \\
\omega_{3}=1-\omega_{1}-\omega_{2}
\end{gathered}
$$

\section{B. Extrapolation}

n

The value of extrapolated data equals to the known point closest to it. 


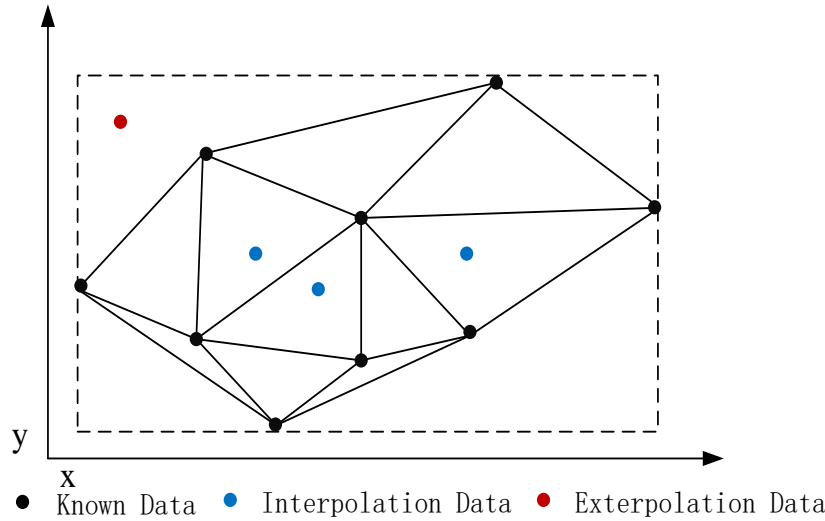

Figure 4. Illustration of interpolation and extrapolation data.

\section{EXPERIMENT AND EVALUATION}

\subsection{Experiment step}

The experiment is conducted in a shopping mall with enough WiFi signals. The test area is approximately $30000 \mathrm{~m}^{2}$. The distance between each reference point (RP) is $6 \mathrm{~m}$. As shown in figure 5 , there are 55 RPs. The Wi-Fi RSS are collected by Honor KIWAL10 smartphone and the signal sampling frequency is $1 \mathrm{~Hz}$. For each RSS, the RSS collection at each RP lasted for 2 minutes.

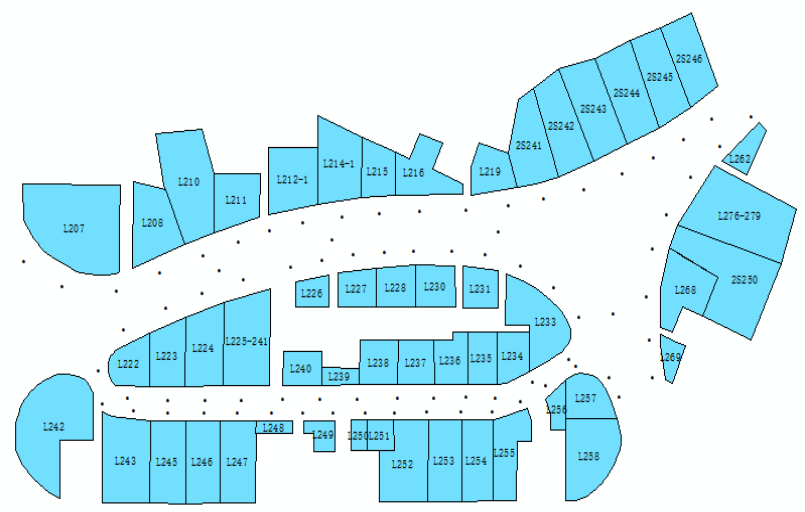

Figure 5. Location of RPs.

Wi-Fi signal distribution in the test environment is shown in Figure 6 . The $\mathrm{x}$ - and $\mathrm{y}$-axis indicate the relative coordinate, and the $\mathrm{z}$-axis shows the number of Wi-Fi signal.

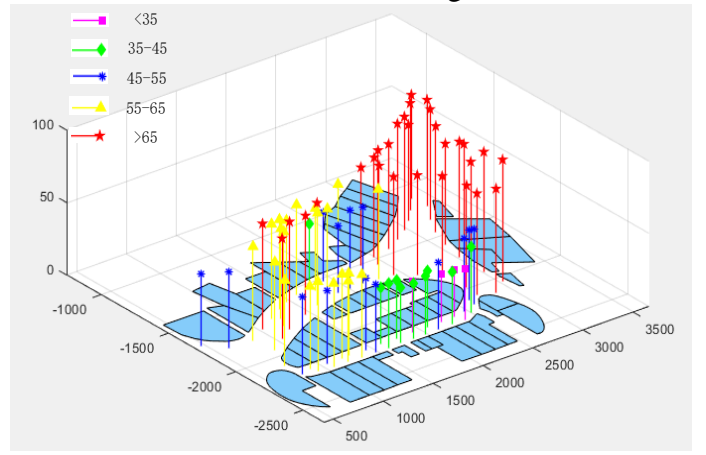

Figure 6. Wi-Fi number distribution of RPs.

The near boundary for ROs in the test environment is shown in Figure 7. The red line region in figure $7 \mathrm{a}$ is the near boundary of L207. Figure $7 \mathrm{~b}$ shows all the near boundary of the ROs.
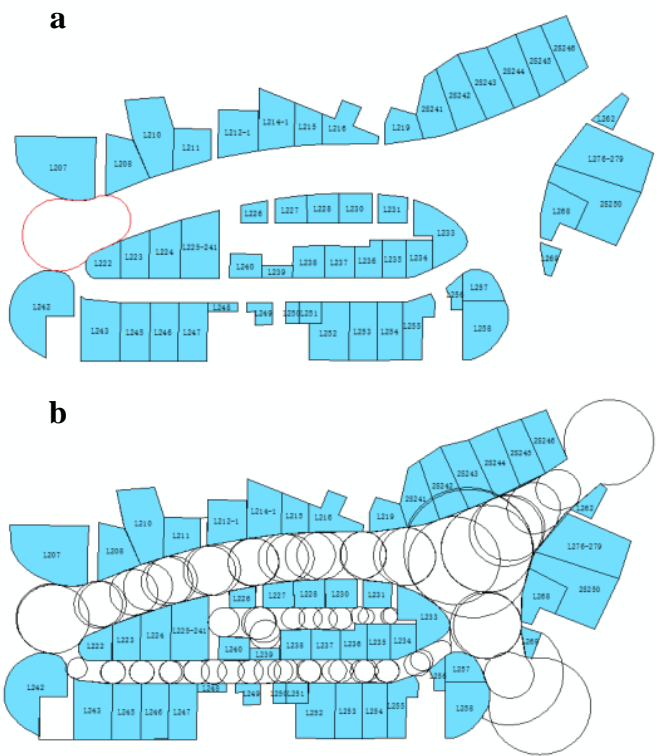

Figure 7. Near boundary of ROs.

\subsection{Localization Results and Evaluation}

10 participants are selected to walk around the test experiment and asked to select the near RO to describe their positioning. Different people may have different choices. The participants include 4 males and 6 females, and their age range from 20 to 48 . Their trajectory is shown in Figure 8. We mark numbers for each track point. The fingerprint matching algorithm we used is WKNN, and the parameter $\mathrm{K}$ is 3 .

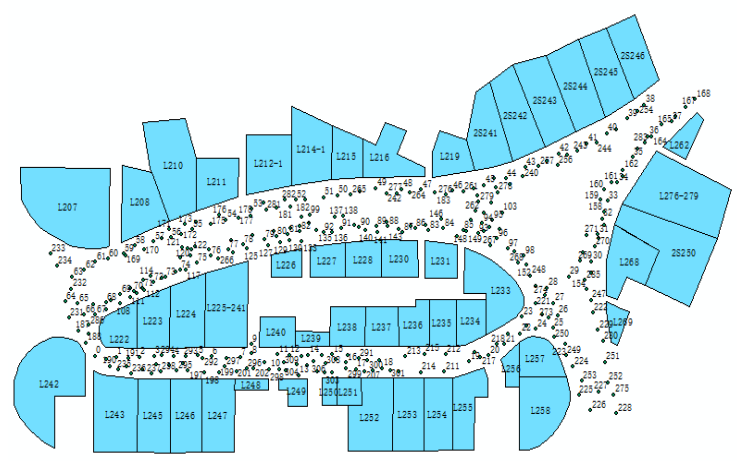

Figure 8. Distribution of track points.

To verify the accuracy of interpolation, when collecting RPs, we add a RP between each RPs. As shown in figure 9, the mean positioning error with non-interpolation and Delaunay-based interpolation density of $3 \mathrm{~m}$ are $6.10 \mathrm{~m}$ and $6.23 \mathrm{~m}$ respectively. The accuracy of the interpolation method can be used to positioning to some extent. The large positioning error comes from fingerprints ambiguity and the location of ambiguity fingerprints is shown in Figure 10. 

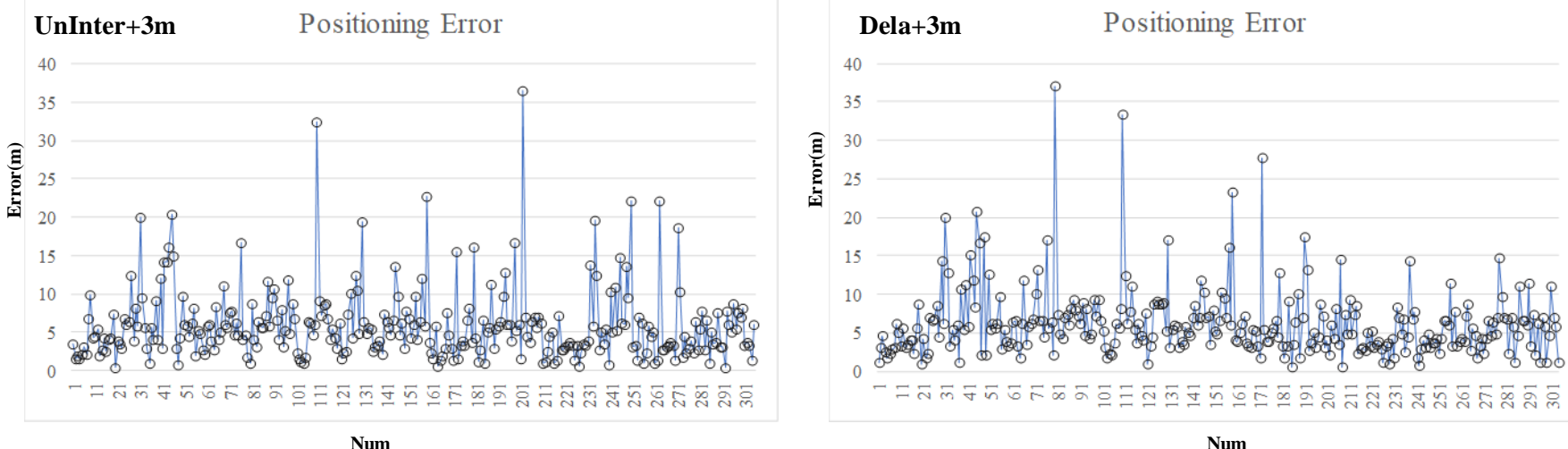

Figure 9. Positioning error with non-interpolation and Delaunay-based interpolation density of $3 \mathrm{~m}$.

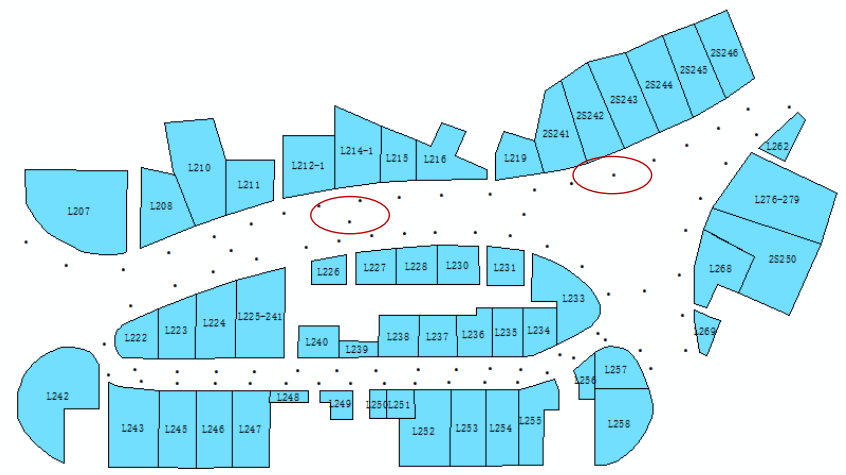

Figure 10. Distribution of ambiguity fingerprints.

Figure 11 shows positioning error with different interpolation methods (IDW and Delaunay) and interpolation density ( $3 \mathrm{~m}$ and $1.5 \mathrm{~m})$ without near relation. The mean error of $\mathrm{IDW}+3 \mathrm{~m}$, IDW $+1.5 \mathrm{~m}$, Dela $+3 \mathrm{~m}$, Dela $+1.5 \mathrm{~m}$ are $6.3 \mathrm{~m}, 5.9 \mathrm{~m}, 6.2 \mathrm{~m}$ and $5.8 \mathrm{~m}$ respectively. IDW $+3 \mathrm{~m}$ represent IDW interpolation method and $3 \mathrm{~m}$ interpolation density.
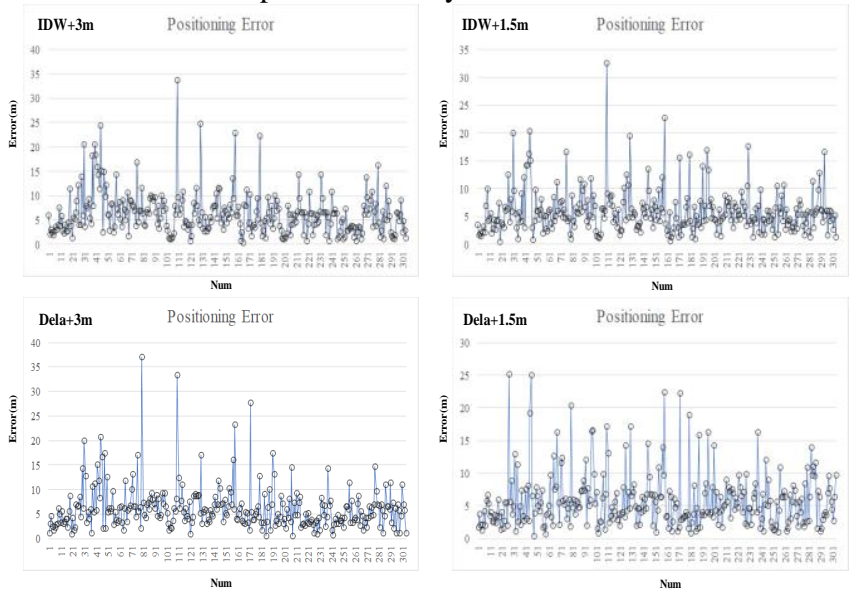

Figure 11. Positioning error with different interpolation methods (IDW and Delaunay) and interpolation density ( $3 \mathrm{~m}$ and $1.5 \mathrm{~m})$ without near relation.

Figure 12 shows positioning error with different interpolation methods and interpolation density considering near relation. The mean error of Near+IDW+3m, Near+IDW+1.5m, Near+Dela $+3 \mathrm{~m}$, Near+Dela $+1.5 \mathrm{~m}$ are $5.0 \mathrm{~m}, 4.2 \mathrm{~m}, 4.3 \mathrm{~m}$ and $3.9 \mathrm{~m}$ respectively. Near+IDW $+3 \mathrm{~m}$ represent IDW interpolation method and $3 \mathrm{~m}$ interpolation density considering near relation.
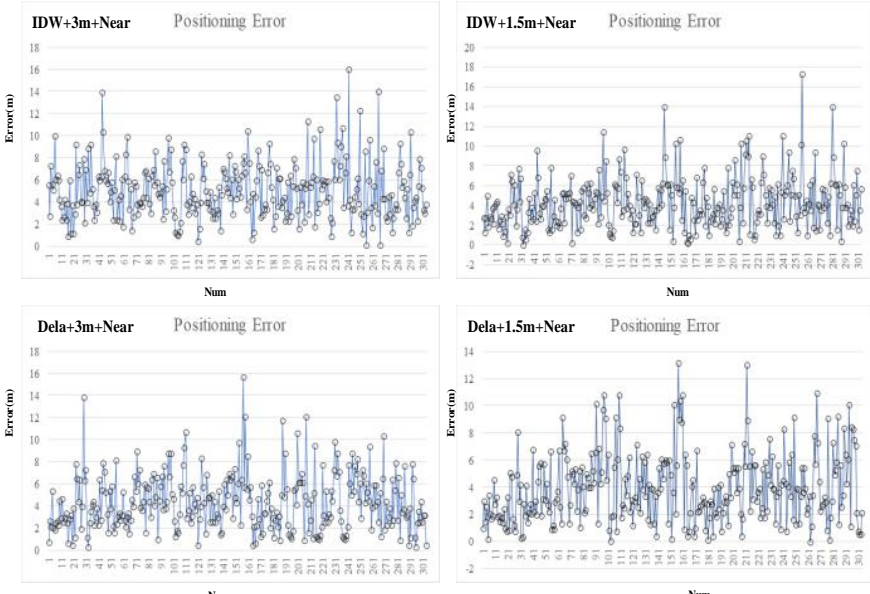

Figure 12. Positioning error with different interpolation methods (IDW and Delaunay) and interpolation density ( $3 \mathrm{~m}$ and $1.5 \mathrm{~m}$ ) considering near relation.

Compared Figure 11 and 12, as interpolation density increases, the positioning accuracy that with near relation or not all improves. As shown in Figure 13, the positioning accuracy is no longer improved when the interpolation density increase to $1 \mathrm{~m}$ and the positioning accuracy is about $3.61 \mathrm{~m}$.

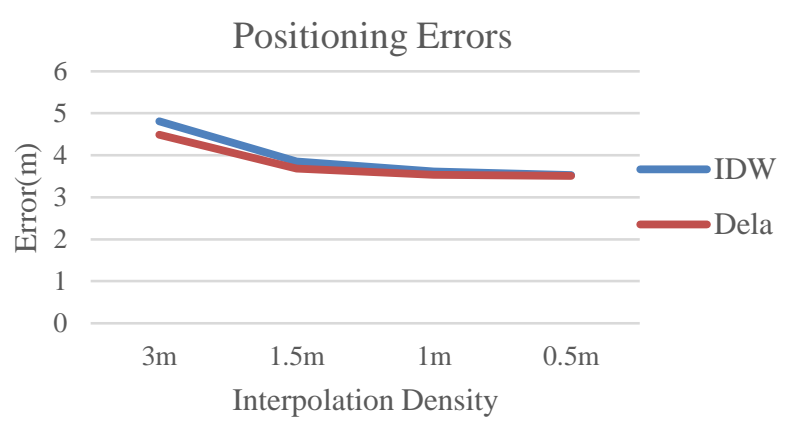

Figure 13. Positioning error of different interpolation methods (IDW and Delaunay) and interpolation density ( $3 \mathrm{~m}$ and $1.5 \mathrm{~m}$ ) considering near relation.

\section{CONCLUSION}

In this paper, we develop a collaborative Wi-Fi fingerprinting indoor positioning method using near relation, which use Delaunay-based interpolation method to encrypt sparse Wi-Fi fingerprint. The interpolated fingerprint is restricted to near boundary, which not only can narrow down the search space of fingerprint but also decrease the RSS ambiguities. The experiment shows that a positioning accuracy of $3.61 \mathrm{~m}$ can be 
achieved with near relation constrain under $1 \mathrm{~m}$ interpolation density. The near boundary provided in this paper is raster-based which has low efficiency. In practical applications, the near boundary should be generated in advance and placed on the server. Additionally, studying other near boundary generation methods and interpolation algorithms (e.g., Kriging) are our future works.

\section{ACKNOWLEDGEMENTS}

Acknowledgements of support for the Funded By Open Research Fund Program of LIESMARS (Grant No. 19P03), National Natural Science Foundation of China (Grant No. 41971341), General Project of the National Natural Science Foundation of Guangdong Province (Grant No. 2019A1515010748, 2019A1515011872) and New Teacher Research Project of Shenzhen University (Grant No. 2019056).

\section{REFERENCES}

B. Huang, Z. Xu, B. Jia, G. Mao, "An online radio map update scheme for Wi-Fi fingerprint-based localization", IEEE Internet of Things Journal, pp. 1-1, 2019.

Chen, Guoliang; Meng, Xiaolin; Wang, Yunjia; Zhang, Yanzhe; Tian, Peng; Yang, Huachao. 2015. "Integrated Wi-Fi/PDR/Sma rtphone Using an Unscented Kalman Filter Algorithm for 3D In door Localization." Sensors 15, no. 9: 24595-24614.

F. Zafari et al., "A Survey of Indoor Localization Systems and T echnologies", ArXiv preprint, 2017.

G. Shen, D. Han and P. Liu, "A Sparse Manifold Learning Appr oach to Robust Indoor Positioning Based on Wi-Fi RSS Fingerp rinting," IEEE Access, vol. 7, pp. 130791-130803, 2019.

Han Xu, Zheng Yang, Zimu Zhou, Longfei Shangguan, Ke Yi, a nd Yunhao Liu. 2015. Enhancing Wi-Fi-based localization with visual clues. In Proceedings of the 2015 ACM International Join t Conference on Pervasive and Ubiquitous Computing (UbiCom p '15). Association for Computing Machinery, New York, NY, USA, 963-974.

DOI:https://doi.org/10.1145/2750858.2807516

Jan, Shau-Shiun; Yeh, Shuo-Ju; Liu, Ya-Wen. 2015. "Received Signal Strength Database Interpolation by Kriging for a Wi-Fi I ndoor Positioning System." Sensors 15, no. 9: 21377-21393.

J. Hu, D. Liu, Z. Yan, H. Liu, " Experimental analysis on weight $\mathrm{K}$-nearest neighbor indoor fingerprint positioning ", IEEE Inter net Things J., vol. 6, no. 1, pp. 891-897, Feb. 2018.

J. Talvitie, M. Renfors, E. S. Lohan, "Distance-based interpolati on and extrapolation methods for RSS-based localization with in door wireless signals", IEEE Trans. Veh. Technol., vol. 64, no. 4, pp. 1340-1353, Apr. 2015.

J. Zuo, S. Liu, H. Xia, Y. Qiao, "Multi-phase fingerprint map ba sed on interpolation for indoor localization using iBeacons", IEE E Sensors J., vol. 18, no. 8, pp. 3351-3359, Jan. 2018.

Leca, Cristian-Liviu; Nicolaescu, Ioan; Ciotirnae, Petrica. 2020. "Crowdsensing Influences and Error Sources in Urban Outdoor Wi-Fi Fingerprinting Positioning." Sensors 20, no. 2: 427.
Liu, Mengyun; Chen, Ruizhi; Li, Deren; Chen, Yujin; Guo, Gua ngyi; Cao, Zhipeng; Pan, Yuanjin. 2017. "Scene Recognition for Indoor Localization Using a Multi-Sensor Fusion Approach." $S$ ensors 17, no. 12: 2847 .

Li, Guoquan; Geng, Enxu; Ye, Zhouyang; Xu, Yongjun; Lin, Jin zhao; Pang, Yu. 2018. "Indoor Positioning Algorithm Based on the Improved RSSI Distance Model." Sensors 18, no. 9: 2820.

L. Mainetti, L. Patrono, I. Sergi, "A survey on indoor positionin g systems", Proc. 22nd Int. Conf. Softw. Telecommun. Comput. Netw. (SoftCOM), pp. 111-120, 2014.

Lohan, Elena S.; Torres-Sospedra, Joaquín; Leppäkoski, Helena; Richter, Philipp ; Peng, Zhe; Huerta, Joaquín. 2017. "Wi-Fi Cro wdsourced Fingerprinting Dataset for Indoor Positioning." Dat $a 2$, no. $4: 32$.

M. Lee, D. Han, "Voronoi Tessellation Based Interpolation Met hod for Wi-Fi Radio Map Construction", IEEE Comm. Letters, v ol. 16, no. 3, pp. 404-407, 2012.

Mozamir M S, Bakar R B A, Din W I S W. Indoor localization e stimation techniques in wireless sensor network: a review, 2018 IEEE International Conference on Automatic Control and Intell igent Systems (I2CACIS). IEEE, 2018: 148-154.

Ren, J., Wang, Y., Niu, C., Song, W., Huang, S. "A Novel Clust ering Algorithm for Wi-Fi Indoor Positioning." IEEE Access 7 (2019): 122428-122434.

R. F. Brena, J. P. García-Vázquez, C. E. Galván-Tejada, D. Muñ oz-Rodriguez, C. Vargas-Rosales, J. Fangmeyer, "Evolution of i ndoor positioning technologies: A survey", J. Sensors, vol. 2017, Mar. 2017.

S. Subedi, H.-S. Gang, N. Y. Ko, S.-S. Hwang, J.-Y. Pyun, "Imp roving indoor fingerprinting positioning with affinity propagatio $\mathrm{n}$ clustering and weighted centroid fingerprint", IEEE Access, vo 1. 7, pp. 31738-31750, 2019.

S. Han et al., "Indoor Localization with a Single Wi-Fi Access P oint Based on OFDM-MIMO", IEEE Systems J., vol. 13, no. 1, pp. 964-72, Mar. 2019.

Tang, H., Xue, F., Liu, T. et al. Indoor Positioning Algorithm Fu sing Multi-Source Information. Wireless Pers Commun 109, 254 $1-2560$ (2019).

V. Moghtadaiee, S. A. Ghorashi and M. Ghavami, "New Recons tructed Database for Cost Reduction in Indoor Fingerprinting Lo calization," IEEE Access, vol. 7, pp. 104462-104477, 2019.

Wang, X, Wei, X, Liu, Y. Received signal strength-based locali zation for large space indoor environments. Int J Distrib Sens N 2017; 13(1): 1-12.

X. Chen, S. Zou, "Improved Wi-Fi indoor positioning based on particle swarm optimization", IEEE Sens. J., vol. 17, no. 21, pp. 7143-7148, Nov. 2017.

X. Du, K. Yang, "A map-assisted Wi-Fi AP placement algorith $\mathrm{m}$ enabling mobile device's indoor positioning", IEEE Syst. J., v ol. 11, no. 3, pp. 1467-1475, Sep. 2017. 
X. Ge, Z. Qu, "Optimization WI-FI indoor positioning KNN alg orithm location-based fingerprint", Proc. IEEE 7th Int. Conf. So ftw. Eng. Service Sci. (ICSESS), pp. 135-137, Aug. 2016.

Xia, Shixiong; Liu, Yi; Yuan, Guan; Zhu, Mingjun; Wang, Zhao hui. 2017. "Indoor Fingerprint Positioning Based on Wi-Fi: An Overview." ISPRS Int. J. Geo-Inf. 6, no. 5: 135.

Y. Li, Y. Zhuang, H. Lan, X. Niu, N. El-Sheimy, "A profile-mat ching method for wireless positioning", IEEE Commun. Lett., vo 1. 20, no. 12, pp. 2514-2517, Dec. 2016. 